\title{
ANÁLISE DA CRISTALINIDADE DOS FLUXANTES ATRAVÉS DO USO DA MICROSCOPIA ÓPTICA DE LUZ TRANSMITIDA*
}

\section{Resumo}

Igor Teixeira da Costa ${ }^{1}$ Letícia Miranda Alves ${ }^{2}$ Anderson Pereira Martins ${ }^{3}$ Hiury Voltz $z^{4}$

Camila Simon Christ ${ }^{5}$ Cássio Santos de Carvalho ${ }^{6}$ Estéfano Aparecido Vieira ${ }^{7}$

Os pós fluxantes são formados por escórias sintéticas que em contato com o aço líquido deve fundir e gerar uma escória líquida. Suas principais funções são: lubrificar e controlar a transferência de calor entre a casca sólida do metal e o molde. Eles possuem composições diferentes para diferentes condições de lingotamento, sendo formados, basicamente, por $\mathrm{CaO}, \mathrm{SiO}_{2}, \mathrm{Al}_{2} \mathrm{O}_{3}, \mathrm{Na}_{2} \mathrm{O}, \mathrm{CaF}_{2}$ e podem conter $\mathrm{MgO}, \mathrm{Li}_{2} \mathrm{O}, \mathrm{K}_{2} \mathrm{O}$ e $\mathrm{B}_{2} \mathrm{O}_{3}$. Como alguns componentes influenciam diretamente na cristalinidade dos fluxantes, como - $\mathrm{CaO}$, o $\mathrm{MgO}$, o $\mathrm{CaF}_{2}$, neste trabalho serão analisadas quatro misturas de pós fluxantes com leves variações na composição de modo que possa gerar cristalinidades diferentes. Para esta análise será utilizada o método de lâminas delgadas, com auxílio de um microscópio óptico de luz transmitida, que tem apresentado resultados bem satisfatório sobre o detalhamento da cristalinidade dos materiais.

Palavras-chave: Pós Fluxantes; Lâminas Delgadas; Microscópio óptico de luz transmitida; Cristalinidade.

\section{ANALYSIS OF CRYSTALLINITY OF MOULD FLUXES THROUGH THE USE OF TRANSMITTED LIGHT OPTIC MICROSCOPY}

\section{Abstract}

The mould fluxes are formed by synthetic slag that in contact with liquid steel ought to merge and generate liquid slag. Its main functions are: lubricate and control the heat transfer between metal shell and mould. They have different compositions for different casting conditions, being formed basically with $\mathrm{CaO}, \mathrm{SiO}_{2}, \mathrm{Al}_{2} \mathrm{O}_{3}, \mathrm{Na}_{2} \mathrm{O}, \mathrm{CaF}_{2}$ and could contain $\mathrm{MgO}, \mathrm{Li}_{2} \mathrm{O}, \mathrm{K}_{2} \mathrm{O}$ e $\mathrm{B}_{2} \mathrm{O}_{3}$. As some components directly influence at the crystallinity of mould fluxes, for example $\mathrm{CaO}, \mathrm{MgO}$ and $\mathrm{CaF}_{2}$, in this paper will be analyzed four mixtures of mould fluxes with slight variations in its composition to generate different crystallinity. For this analysis will be used the solid slag film method with aid of a transmitted light optical microscope, which has presented satisfactory results on the detailing of fluxes crystallinity.

Keywords: Mould fluxes; Solid slag film; Transmitted light optical microscope; Crystallinity.

1 Engenheiro Mecânico, Mestrando em Engenharia Metalúrgica e de Materiais do Programa de PósGraduação em Engenharia Metalúrgica e de Materiais - PROPEMM, Instituto Federal do Espírito Santo - IFES, Vitória, ES, Brasil.

2 Engenheira Metalúrgica, Mestre em Engenharia Metalúrgica e Materiais, IFES, Vitória - ES, Brasil.

3 Técnico Metalúrgico, Técnico de laboratório/ metalurgia, IFES, Vitória, ES, Brasil.

4 Engenheiro Metalúrgico, Mestrando em Engenharia Metalúrgica e de Materiais do Programa de PósGraduação em Engenharia Metalúrgica e de Materiais - PROPEMM, IFES, Vitória, ES, Brasil.

5 Engenheira Metalúrgica, Mestranda em Engenharia Metalúrgica e de Materiais do Programa de PósGraduação em Engenharia Metalúrgica e de Materiais - PROPEMM, IFES, Vitória, ES, Brasil.

6 Bolsista de Pós-Doutorado, Programa de mestrado em metalurgia e materiais, IFES, Vitória - ES, Brasil

7 Engenheiro Metalúrgico, Prof. Dr. Professor do Programa de Mestrado em Engenharia Metalúrgica e Materiais, IFES, Vitória, ES, Brasil. 


\section{INTRODUÇÃO}

Os pós fluxantes são escórias sintéticas constituídos basicamente pelo sistema $\mathrm{CaO}$ $\mathrm{SiO}_{2}-\mathrm{Al}_{2} \mathrm{O}_{3}-\mathrm{Na}_{2} \mathrm{O}-\mathrm{CaF}_{2}$ e podem conter $\mathrm{MgO}, \mathrm{Li}_{2} \mathrm{O}, \mathrm{K}_{2} \mathrm{O}$ e $\mathrm{B}_{2} \mathrm{O}_{3}$ [1]. Eles são continuamente adicionados no topo do molde onde gradualmente são fundidos a uma temperatura em torno de 1040 a $1300^{\circ} \mathrm{C}$ e geram uma escória líquida com características específicas para cada condição de lingotamento [2]. Essa escória infiltra-se no gap formado entre o molde e a casca de metal solidificada e sofre um resfriamento devido a água que continuamente resfria o molde. Portanto, é gerada uma película de escória sólida (aproximadamente $2 \mathrm{~mm}$ de espessura) seguida de uma fina camada líquida (aproximadamente $0,2 \mathrm{~mm}$ de espessura) contra a casca [3, 4].

As camadas de escória, líquida e sólida, são responsáveis pelo controle das duas principais funções do pó fluxante: lubrificação e transferência de calor horizontal entre molde e casca $[3,4,5]$. Além disso, os fluxantes absorvem inclusões do aço, permitem isolamento térmico e protegem a superfície contra a oxidação $[1,4,5]$.

O controle da transferência de calor horizontal entre casca e molde é fundamental para aumentar consideravelmente a probabilidade de obter um produto final sem defeitos e com boa qualidade [1, 2, 3, 4, 5] além de evitar problemas de operação [2]. A principal propriedade do pó fluxante que influencia neste controle é a cristalinidade [4]. Quanto maior for a porcentagem de fase cristalina na camada solidificada, menor será a taxa de transferência de calor por radiação entre molde e placa. [2, 3, 4, 5].

Para cada tipo de aço é utilizado um tipo diferente de pó fluxante que devido a mudança na composição química afeta a cristalinidade. Em condições onde são necessários fluxantes que permitam alta transferência de calor entre casca/molde, como é o caso dos aços baixo carbono, o pó fluxante deve ter a cristalinidade reduzida, ou seja, ter predominância de fase vítrea na camada solidificada. Enquanto que para baixa transferência de calor placa/molde, caso dos aços peritéticos, o pó deve ser mais cristalino, ou seja, ter predominância de fases cristalinas [2, 3].

A petrologia é o ramo da Geologia que estuda as rochas, que são formadas no geral pela associação de minerais [6]. Ela utiliza a microscopia óptica de transmissão para identificação desses minerais e as amostras, na maioria dos casos, são preparadas pelo método de lâminas delgadas $[7,8]$. As lâminas são obtidas através de uma seção extremamente fina do material, em torno de $0,03 \mathrm{~mm}$, conforme mostrado na figura 1 . Tem como principal vantagem o fato de todos os cristais e/ou minerais presentes na seção possuírem uma mesma espessura já conhecida o que permite a determinação de uma série de propriedades ópticas, em especial, àquele referente à interferência da luz [9].

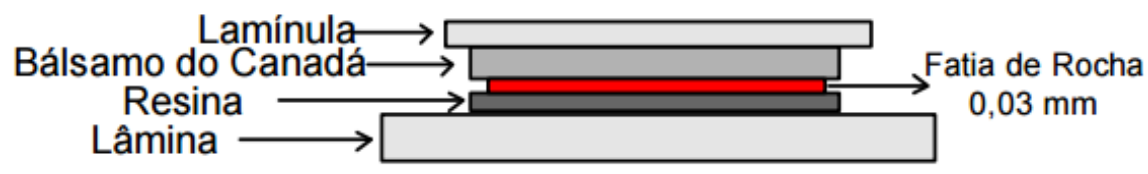

Figura 1. Representação esquemática de uma montagem em lâmina de um mineral granulado [9]. 
A espessura da lâmina é uma etapa muito importante pois garante a passagem de luz pelos minerais translúcidos, que através de técnicas como a polarização, ângulo de extinção, hábito, birrefringência e relevo possibilitam sua caracterização [10]. A proposta deste trabalho é mostrar a eficiência deste método na detecção da cristalinidade em diversos fluxantes, assim como a análise dos grãos de cada um.

\section{MATERIAIS E MÉTODOS}

O presente estudo utilizou a microscopia óptica de transmissão e o método de lâminas delgadas para a identificação de cristais em 4 diferentes fluxantes cuja composição química é mostrada na tabela 1 do item 3. Os fluxantes P1, P2 e P3 foram formulados com base em resíduos de mármore e granito, variando principalmente os componentes $\mathrm{CaO}, \mathrm{F}$ e $\mathrm{MgO}$ [12]. Já o PC é um fluxante peritético comercial obtido de uma indústria siderúrgica.

\subsection{Preparação das amostras}

A preparação das amostras seguiu a metodologia do "Teste de Stollberg modificado" [3]. Os fluxantes foram descarbornetados a temperatura de $650^{\circ} \mathrm{C}$ por 16 horas ao ar, e então foram colocadas $30 \mathrm{~g}$ dessas amostras em um cadinho de alumina e aquecidas a uma temperatura de $1300^{\circ} \mathrm{C}$ durante 20 minutos. Após a fusão, as amostras foram colocadas num cadinho de aço inoxidável e aquecidas por 20 minutos numa temperatura de $610^{\circ} \mathrm{C}$. Para o resfriamento, foi realizada uma têmpera em água no qual metade do cadinho foi imerso neste fluido.

A etapa de descarbonetação foi necessária para eliminação dos compostos voláteis e consequente diminuição da evaporação destes componentes na escória líquida de fluxante durante o aquecimento [11]. $\mathrm{O}$ aquecimento a $1300^{\circ} \mathrm{C}$ permite que o fluxante esteja totalmente fundido durante o processo[5]. Já o recozimento na temperatura de $610^{\circ} \mathrm{C}$ teve como objetivo a indução da cristalização do material [3].

\subsubsection{Confecção das lâminas}

As lâminas foram confeccionadas seguindo padrões da ABNT/NBR [9]. Após o resfriamento, a seção transversal das escórias foi embutida em uma resina acrílica para análise do crescimento dos grãos desde a parte em contato com o cadinho até a superfície das amostras. Posteriormente foram lixadas e polidas para que houvesse nivelamento da base e um bom acabamento. Em seguida foram coladas em lâminas de vidro onde foram novamente lixadas e polidas para que atingissem a espessura desejada de aproximadamente $0,3 \mathrm{~mm}$. Esta operação pode ser simplificada em 4 etapas:

1) Embutimento: Foi realizado embutimento a frio com resina acrílica na seção transversal das escórias para que houvesse uma maior superfície de contato da amostra com a lâmina na etapa de colagem. Isso garantiu o não desprendimento da amostra.

2) Lixamento/Polimento: Foram utilizadas lixas de alumina com granulometrias de $400,600,800,1000$ e 1200 mesh e o polimento foi feito com o auxílio de pasta de abrasivo de diamante $1 \mu$ e água. Utilizou-se a politriz Arotec® modelo Aropol 
VV, pertencente ao laboratório de metalografia do IFES, campus Vitória. Figura $1 \mathrm{a}$.

3) Colamento: A amostra e a lâmina de vidro foram levemente aquecidas para a inserção da cola Araldite entre elas. $O$ trio foi pressionado para garantir uma boa fixação isenta de bolhas. Figura $1 b$.

4) Corte: Após o colamento, as amostras foram submetidas a um corte de espessura inferior a $2 \mathrm{~mm}$. Para isso, foi utilizada uma máquina Cortadora Metalográfica de Pequena Dimensão, marca Militon, pertencente ao laboratório de Engenharia de Superfícies do IFES, campus Vitória.

5) Lixamento/Polimento Final: Foram utilizados os mesmos equipamentos da etapa 2. Para o lixamento, iniciou-se o procedimento com a lixa de 400 mesh até que a amostra atingisse uma espessura de $0,40 \mathrm{~mm}$; posteriormente a lixa de 600 até a espessura de 0,10 a 0,12mm; em seguida a lixa de 1000 até uma espessura de 0,08mm e por fim, a de 1200 até a espessura de 0,045 a $0,05 \mathrm{~mm}$. Para o polimento, pasta de abrasivo de diamante $1 \mu$ e água foram utilizados até a obtenção da espessura final de $0,03 \mathrm{~mm}$. Figura 1c.
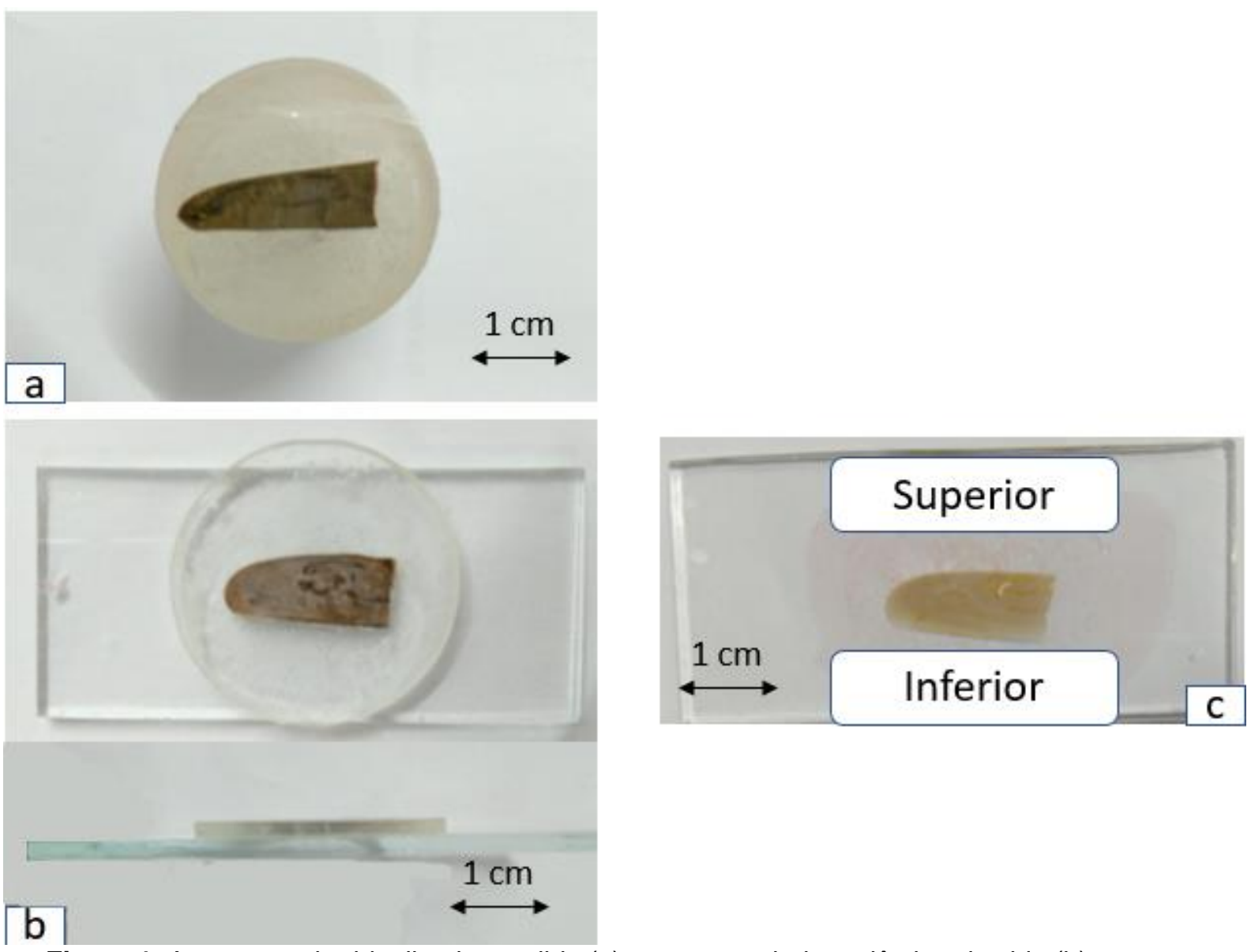

Figura 1. Amostra embutida, lixada e polida (a); amostra colada na lâmina de vido (b); amostra cortada, lixada e polida, pronta para análise (c).

Após o procedimento de preparação das lâminas, foi analisado as propriedades ópticas das amostras por meio de microscópio petrográfico de luz transmitida, Leica DM750, pertencente ao IFES, campus Vitória. As lâminas foram avaliadas mediante um compensador de 530nm e sob auxílio do software Laz EZ para obtenção das imagens. 
Para melhor entendimento do resultado obtido no microscópio, foi feita uma ilustração (Figura 2) dos aspectos cristalino e amorfo que são visualizados com nicois cruzados e utilizando o compensador de 530nm.

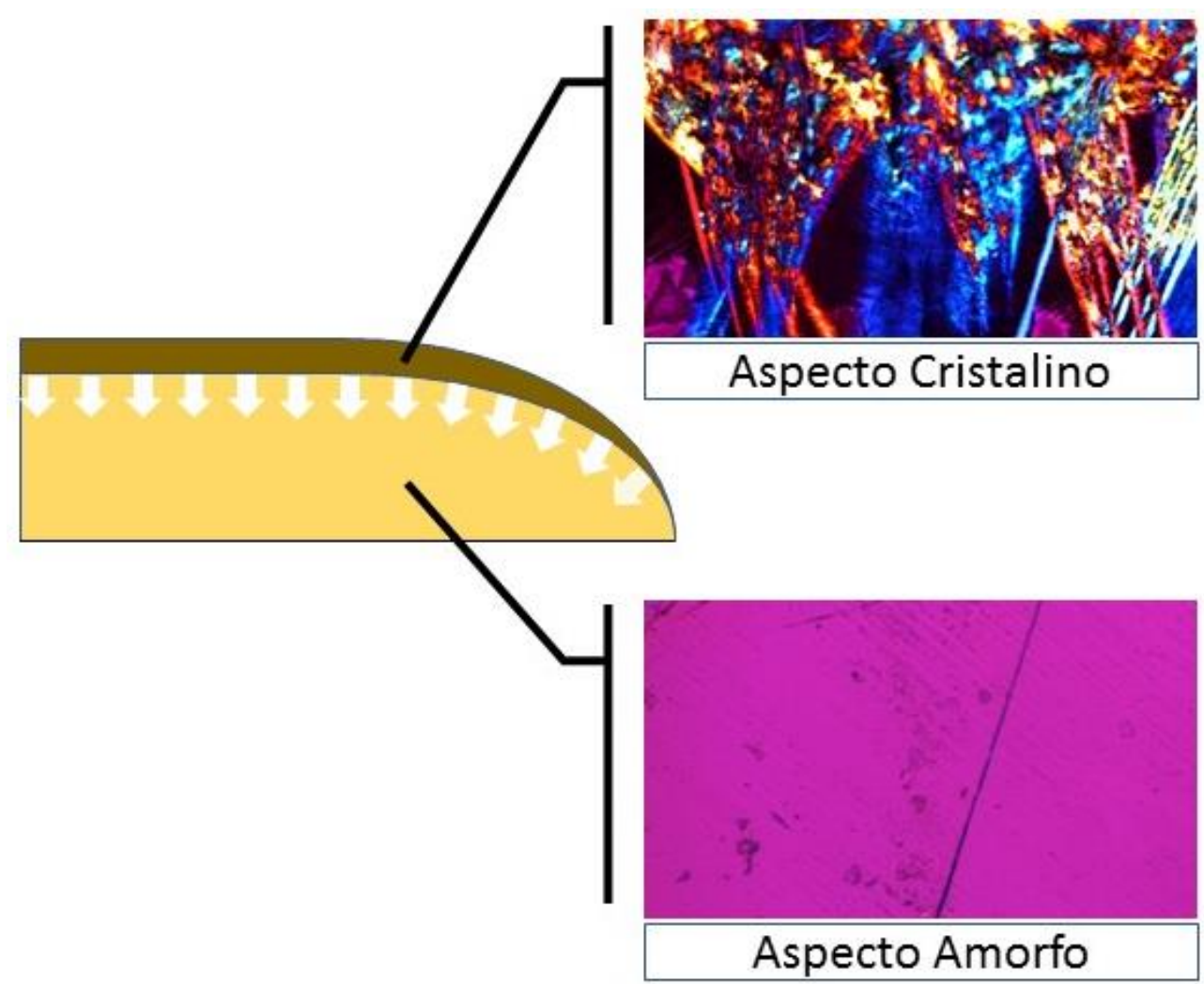

Figura 2. Representação esquemática dos aspectos (cristalino e amorfo) obtidos pelo microscópio Leica DM750 com nicois cruzados e utilizando um compensador de $530 \mathrm{~nm}$.

\section{RESULTADOS E DISCUSSÃO}

Os resultados da tabela 1 foram obtidos por meio de balanço de massa a partir de análises obtidas por espectroscopia por energia dispersiva (EDS). As análises foram feitas em um equipamento da Marca Oxford acoplado a um microscópio eletrônico de varredura (MEV) da Marca Zeiss Mod. Evo 10 pertencente ao Laboratório de Microscopia Eletrônica de Varredura do IFES, campus Vitória. As composições obtidas podem ter margem de erro em torno de $5 \%$, especialmente para elementos de menor concentração, no entanto os resultados estão de acordo com o esperado pois maiores concentrações de $\mathrm{CaO}$ e menores de $\mathrm{MgO}$ foram identificadas a medida que os fluxantes se tornam mais cristalinos, ou seja, na ordem da esquerda para direita, P1 a PC. 
Tabela 1. Composição química de fluxantes realizados por meio de resíduos de granito e mármore (P1, P2, P3) provenientes de Alves e colaboradores (2016) e do fluxante comercial peritético (PC) via balanço de massa a partir dos resultados de EDS [12].

\begin{tabular}{lcccc}
\hline Componentes & P1 & P2 & P3 & PC \\
\hline $\mathrm{CaO}$ & $34,1 \%$ & $35,6 \%$ & $44 \%$ & $44,1 \%$ \\
\hline $\mathrm{SiO}_{2}$ & $25,1 \%$ & $23,6 \%$ & $24 \%$ & $30,1 \%$ \\
\hline $\mathrm{F}$ & $10,4 \%$ & $12,3 \%$ & $10,6 \%$ & $8,0 \%$ \\
\hline $\mathrm{Al}_{2} \mathrm{O}_{3}$ & $5,3 \%$ & $4,9 \%$ & $5,2 \%$ & $3,9 \%$ \\
\hline $\mathrm{MgO}$ & $8,9 \%$ & $8,2 \%$ & $2,9 \%$ & $0,9 \%$ \\
\hline $\mathrm{Na}_{2} \mathrm{O}$ & $4,8 \%$ & $4,5 \%$ & $4,9 \%$ & $4,9 \%$ \\
\hline $\mathrm{K}_{2} \mathrm{O}$ & $1,6 \%$ & $1,5 \%$ & $1,6 \%$ & $0,3 \%$ \\
\hline $\mathrm{Fe}_{2} \mathrm{O}_{3}$ & $1,7 \%$ & $1,6 \%$ & $1,7 \%$ & $1,6 \%$ \\
\hline $\mathrm{Li}_{2} \mathrm{O}$ & $0 \%$ & $0 \%$ & $0 \%$ & $0 \%$ \\
\hline $\mathrm{C}_{\text {total }}$ & $8,3 \%$ & $7,8 \%$ & $5 \%$ & $6,2 \%$ \\
\hline $\mathrm{C}_{\text {livre }}$ & $1,9 \%$ & $1,8 \%$ & $2,1 \%$ & - \\
\hline $\mathrm{Basicidade}$ & 1,71 & 1,86 & 1,83 & 1,47 \\
$\left(\mathrm{CaO} / \mathrm{SiO}_{2}\right)$ & & & & \\
\hline
\end{tabular}

Após o Teste de Stollberg modificado, as amostras obtiveram o aspecto mostrado na figura 3. Aparentemente, pode ser observado a diminuição da translucidez e o aumento da resistência à transferência de calor horizontal das amostras de P1 a PC. Portanto, à medida que o teor de $\mathrm{MgO}$ diminui e teor de $\mathrm{CaO}$ aumenta têm-se um aumento da cristalinidade aparente. O F também é um fator determinante para 0 aumento da cristalinidade, principalmente se comparado o P1 com o P2, que tem $10,4 \%$ e $12,3 \%$ respectivamente, pois o $\mathrm{CaO}$ e o $\mathrm{MgO}$ não variam de forma significativa. No entanto, para confirmação microscópica do que pode ser percebido macroscopicamente foi realizado lâminas das escórias (Figura 4) para então serem analisadas no Microscópio de Luz Transmitida. 

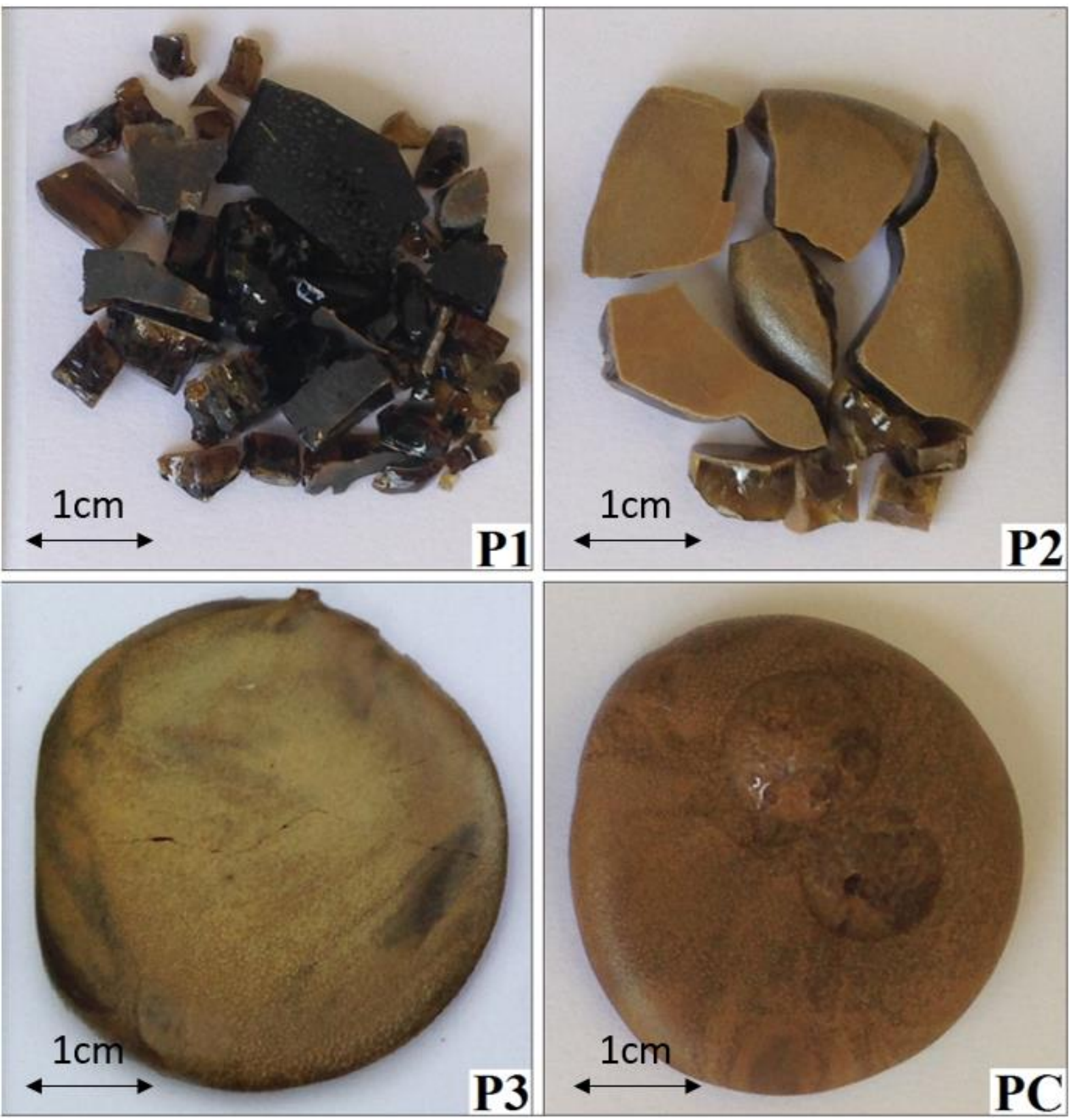

Figura 3. Aspecto macroscópico dos fluxantes após Teste de Stollberg Modificado.

A figura 4 mostra as micrografias das lâminas observadas no microscópio de luz transmitida, a qual se referem à superfície das escórias (lado oposto ao cadinho). Nela podem ser observadas, de P1 a P3, o aumento gradativo da camada cristalina com a variação de $\mathrm{F}, \mathrm{CaO}$ e $\mathrm{MgO}$ assim como pode ser percebido na figura 2. A amostra P1 - contendo $34,1 \% \mathrm{CaO}, 10,4 \% \mathrm{~F}$ e $8,9 \% \mathrm{MgO}$ - apresenta uma camada cristalina de aproximadamente $200 \mu$, enquanto que P2 com uma leve redução de $\mathrm{MgO}$ e um aumento de 1,5\% de $\mathrm{CaO}$ e 1,9\% de $\mathrm{F}$ já apresentou um aumento da cama cristalina, apresentando aproximadamente $600 \mu$. A amostra P3 já contém uma composição mais próxima do fluxante peritético comercial (PC), que comparado com P2, mesmo contendo menos $\mathrm{F}$, o aumento considerável de $\mathrm{CaO}$ e a redução drástica de $\mathrm{MgO}$ acarretaram numa total cristalização do material, assim como o fluxante peritético comercial (PC). 
É importante destacar as diferenças morfológicas entre os fluxantes fabricados por meio de resíduos (P1, P2 e P3) e aquele obtido comercialmente (PC). Neste os grãos possuem um aspecto acicular, enquanto que naqueles o aspecto é axial. Não se sabe ao certo o que influencia nessa diferença de aspecto, uma vez que apesar de possuir uma composição próxima ao fluxante peritético comercial, as amostras providas dos resíduos de mármore e granito possuem uma composição mais baixa de $\mathrm{SiO}_{2}$ e maior concentração de $\mathrm{MgO}$ e $\mathrm{Al}_{2} \mathrm{O}_{3}$. Além disso é necessária uma análise prática da influência dessa diferença de morfologia na resistência ao fluxo de calor horizontal.
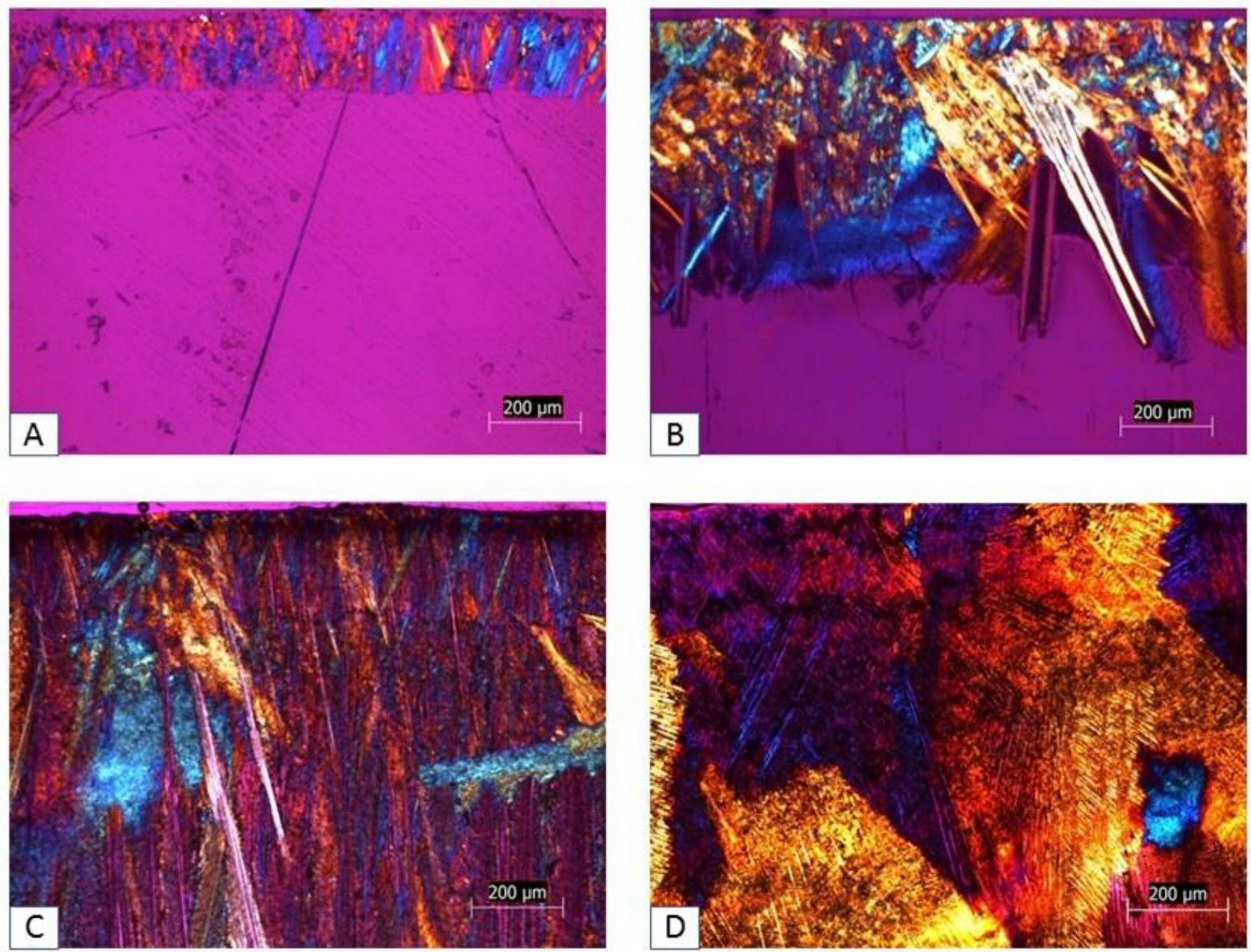

Figura 4. Micrografias obtidas no microscópio de luz transmitida após preparação das amostras pelo método de lâminas delgadas com nicois cruzado e utilizando um compensador de $530 \mathrm{~nm}$. Na sequência estão o fluxante P1 (A), P2 (B), P3 (C) e PC (D).

\section{CONCLUSÃO}

A utilização do método de lâminas delgadas juntamente com o auxílio do microscópio óptico de luz transmitida, seguindo a metodologia utilizada na petrografia para descrição de minerais e rochas já havia apresentados resultados favoráveis e promissores na caracterização de pós fluxantes [10,12]. Contudo, ela apresentou resultados muito satisfatórios na análise da cristalinidade dos pós fluxantes e dos contornos de grãos.

Neste trabalho, conseguiu-se analisar o aumento da cristalinidade nos pós fluxantes influenciados pela variação de $\mathrm{F}, \mathrm{CaO}$ e $\mathrm{MgO}$ e a diferença morfológica para os 
fluxantes formados através dos resíduos de mármore e granito - P1, P2 e P3 - para o fluxante peritéticos comercial - PC.

A análise do fator resultante nessa diferença de morfologia deverá ser feita em trabalhos futuros, assim como a influência dessa morfologia na resistência ao fluxo de calor horizontal.

\section{Agradecimentos}

Os autores agradecem ao Instituto Federal de Ciência e Tecnologia do Espírito Santo - IFES pelo apoio, em especial ao Laboratório de Engenharia de Superfície e Desgaste e ao Laboratório de Metalografia. Agradecem também pelo apoio financeiro a CAPES, FINEP, CNPq Proc. 302523/2016-2 e FAPES T.O. 243/2016.

\section{REFERÊNCIAS}

1 Garcia A, Spim JA, Dos Santos CA, Cheung N. Lingotamento contínuo de aços. São Paulo: Associação brasileira de Metalurgia e Materiais, 2006.

2 Li Z, Mills K, Bezerra MCC. Characteristics of mould flux films for casting MC and LC steels. XXXV Seminário de Fusão, Refino e Solidificação dos Metais. Salvador, Brasil. 2004.

3 LI Z, Thackray R, Mills KC. A test to determine crystallinity of mould fluxes. VII International Conference on Molten Slags, Fluxes and Salts. The South African Institute of Mining and Metallurgy. 2004.

4 Mills KC, Fox AB. The role of mould fluxes in continuous castin - so simple yet so complex. ISIJ International. 2003; Vol. 43 (No. 10): 1479-1486.

5 Mills KC, Fox AB, Thackray RP, LI Z. The performance and properties of mould fluxes. VII International on Molten Slags Fluxes and Salts. The South African Institute of Mining and Metallurgy. 2004.

6 Carvalho CS, Martins AP, Alves LM, Voltz H, Junior VM, Lorenzoni CB, Vieira EA. Uso da microscopia de luz transmitida na caracterização de fluxantes. ABMWeek. 2016.

7 Rodríguez P.; Arab P. B.; Celestino T. B.; Characterization of rock cracking patterns in diametral compression tests by acoustic emission and petrographic analysis. International Jornal of Rock Mechanics Sciences. 73-85. 2016.

8 Ribeiro, J.; Ruiz, S, I.; et al. Petrography and mineralogy of self-burning coal wastes from anthracite mining in the el bierzo coalfield (NW spain). International Journal of coal geology. 92-106. 2016.

9 Machado FB, Nardy AJ. Mineralogia Óptica. São Paulo: Oficina de textos, 2016.

10 ASSOCIAÇÃO BRASILEIRA DE NORMAS TÉCNICAS, NBR 15845-1:2015: Rochas para revestimento, análise petrográfica, Rio de Janeiro: (1992).

11 Görnerup M, Hayashi M, Däcker CA, Seetharaman S. Mould fluxes in continuous casting of steel - characterization and performance tuning. VII International Conference on Molten Slags Fluxes and Salts. 2004.

12 Alves LM. Formulação de pó fluxante peritéticos a partir de resíduos de mármore e granito. Dissertação de Mestrado. IFES. 2016. 